\title{
BMJ Open Comparative cardiovascular morbidity and mortality in patients taking different insulin regimens for type 2 diabetes: a systematic review
}

\author{
Hilary I Price, Meghan D Agnew, John-Michael Gamble
}

To cite: Price HI, Agnew MD, Gamble J-M. Comparative cardiovascular morbidity and mortality in patients taking different insulin regimens for type 2 diabetes: a systematic review. BMJ Open 2015;5: e006341. doi:10.1136/ bmjopen-2014-006341

- Prepublication history and additional material is available. To view please visit the journal (http://dx.doi.org/ 10.1136/bmjopen-2014006341).

Received 8 August 2014 Revised 15 February 2015 Accepted 16 February 2015

CrossMark

School of Pharmacy, Memorial University of Newfoundland, St. John's, Newfoundland and Labrador, Canada

Correspondence to Dr John-Michael Gamble; jgamble@mun.ca

\section{ABSTRACT}

Objectives: To summarise the literature evaluating the association between different insulin regimens and the incidence of cardiovascular morbidity and mortality in adults with type 2 diabetes.

Design: Systematic review.

Methods: Multiple biomedical databases (The Cochrane Library, PubMed, EMBASE, and International Pharmaceutical Abstracts) were searched from their inception to February 2014. References of included studies were hand searched. Randomised controlled trials (RCTs), cohort studies or case-control studies examining adults ( $\geq 18$ years) with type 2 diabetes taking any type, dose and/or regimen of insulin were eligible for inclusion in this review.

Outcome measures: Primary outcomes were cardiovascular morbidity and mortality including fatal and/or non-fatal myocardial infarction, fatal and/or nonfatal stroke, major adverse cardiac events and cardiovascular death. All-cause mortality was assessed as a secondary outcome.

Results: Of the 3122 studies identified, 2 RCTs and 6 cohort studies were selected. No case-control studies met the inclusion criteria. The studies examined a total of 109910 patients. Quantitative synthesis of the results from included studies was not possible due to a large amount of clinical heterogeneity. Each study evaluated cardiovascular outcomes across different insulin-exposure contrasts. RCTs did not identify any difference in cardiovascular risks among a fixed versus variable insulin regimen, or a prandial versus basal regimen, albeit clinically important risks and benefits cannot be ruled out due to wide Cls. Findings from cohort studies were variable with an increased and decreased risk of cardiovascular events and all-cause mortality being reported.

Conclusions: This systematic review of randomised and non-randomised studies identifies a substantive gap in the literature surrounding the cardiovascular morbidity and mortality of patients using different regimens of insulin. There is a need for more consistent high-quality evidence investigating the impact of insulin use on cardiovascular outcomes in patients with type 2 diabetes.

Trial registration number: PROSPERO: CRD42014007631.

\section{Strengths and limitations of this study}

- Our systematic review identifies a large knowledge gap in the area of diabetes pharmacotherapy and cardiovascular outcomes.

- We limited our systematic review to include studies designed to evaluate the effect of different insulin regimens on cardiovascular outcomes.

- A quantitative synthesis was not possible due to the large amount of clinical heterogeneity in exposures across studies.

\section{INTRODUCTION}

Clinical practice guidelines recommend insulin therapy for patients with type 2 diabetes unable to reach or maintain their glycaemic targets despite lifestyle and metformin therapy. ${ }^{12}$ The utilisation of insulin has been increasing over time and it has been estimated that $30 \%$ of patients are currently using insulin and up to $86 \%$ of patients with type 2 diabetes will initiate insulin therapy in their lifetime. ${ }^{3-6}$ As the prevalence of insulin use continues to rise it will be increasingly critical to determine the impact of various insulin regimens on patient important outcomes. Despite decades of experience in using insulin, there appears to be a stark lack of evidence regarding the effectiveness of insulin on patient important outcomes in patients with type 2 diabetes. ${ }^{7} 8$ Moreover, there is debate in the scientific community over the relative safety of exogenous insulin within the context of glycaemic management in patients with type 2 diabetes. ${ }^{8} 9$

Given the uncertainty surrounding the safety and effectiveness of insulin, the fact that cardiovascular (CV) disease is the leading cause of death among patients with type 2 diabetes, ${ }^{10}$ and the progressive nature of type 2 diabetes whereby insulin is eventually required to achieve optimal glycaemic control, we were interested in the relative 
effects of different insulin regimens on CV outcomes. To summarise the literature on this topic we conducted a systematic review of randomised controlled trials (RCTs) and observational studies that evaluated the incidence of CV morbidity and mortality in adults with type 2 diabetes taking different insulin regimens.

\section{METHODS}

This study protocol was developed a priori and was registered in the PROSPERO international prospective register of systematic reviews (PROSPERO record: CRD42014007631 (available at http://www.crd.york.ac.uk/ PROSPERO/display_record.asp?ID=CRD42014007631) $){ }^{11}$ Using standard systematic review methods we searched for RCTs, cohort studies or case-control studies that examined the association between different insulin treatment regimens in patients with type 2 diabetes.

\section{Literature search and screening strategy}

A comprehensive search was conducted in the following electronic databases from their inception to February 2014: The Cochrane Library, PubMed, EMBASE and the International Pharmaceutical Abstracts. A combination of MeSH, EMTREE, and free text search terms were employed to identify all RCTs, cohort studies or casecontrol studies designed to evaluate the effect of different insulin regimens on CV outcomes in adults with type 2 diabetes. Studies examining short-term insulin regimens in hospitalised patients were excluded. There were no restrictions on publication date or publication status. Articles were restricted to English language only. A sample search strategy can be found in online supplementary appendix A with the full search strategy available in the PROSPERO protocol.

Citation screening was conduced using standard systematic review methods whereby two independent trained reviewers (HIP and MDA) used standardised inclusion and exclusion criteria. The title and abstract of each citation was screened and a list of articles for full text review was generated. The full texts of the select articles were evaluated for inclusion and the reference lists of included studies were hand searched for relevant articles. In the case of a discrepancy between reviewers, a third reviewer (J-MG) was consulted to reach consensus.

\section{Risk of bias assessment}

The methodological quality of included studies was evaluated. RCTs were assessed using the Cochrane Risk of Bias tool ${ }^{12}$ and cohort studies were assessed using the Newcastle-Ottawa Scale. ${ }^{13}$ Two independent reviewers (HIP and MDA) performed each quality assessment, consulting a third reviewer (J-MG) if necessary.

\section{Data collection and analysis}

Data were extracted independently in duplicate using a standardised and piloted data extraction spreadsheet (HIP and MDA), consulting a third reviewer (J-MG) if necessary to reach consensus. Bibliographic details, study research question(s)/objective(s), study design, number of participants, study duration, intervention(s) (insulin type, dose and regimen), comparison(s) (insulin type, dose and regimen) and relevant quantitative results for the primary and secondary outcomes of interest were extracted.

The primary outcome of interest was the incidence of CV disease including fatal and/or non-fatal myocardial infarction (MI), fatal and/or non-fatal stroke, CV death, and major acute coronary event (MACE) as defined by the studies reviewed. The secondary outcome of interest was all-cause mortality.

Data were analysed using Stata V.12 (StataCorp LP, Stata Statistical Software: Release 12. College Station, Texas, USA). The principal summary measure for RCTs was an unadjusted relative risk (RR) calculated based on the number of CV events and total number of patients in the intervention and comparator groups. The principal summary measures for cohort studies were the adjusted point estimates reported by each study. Unadjusted RRs $(95 \%$ CI) were calculated for cohort studies that did not report point estimates and provided sufficient data. The authors determined that it was inappropriate to meta-analyse the $\mathrm{CV}$ outcome data due to the clinical heterogeneity of insulin regimens (exposure groups) compared across studies. Results are presented stratified by CV event type and study design.

\section{RESULTS}

This systematic literature search identified 3122 records once duplicates were removed (figure 1). Following title and abstract screening the full text of 53 potentially relevant studies were retrieved and evaluated for inclusion. Of these, eight studies met our inclusion criteria. Studies were excluded because they had an ineligible study design $(n=7)$, they did not compare insulin regimens

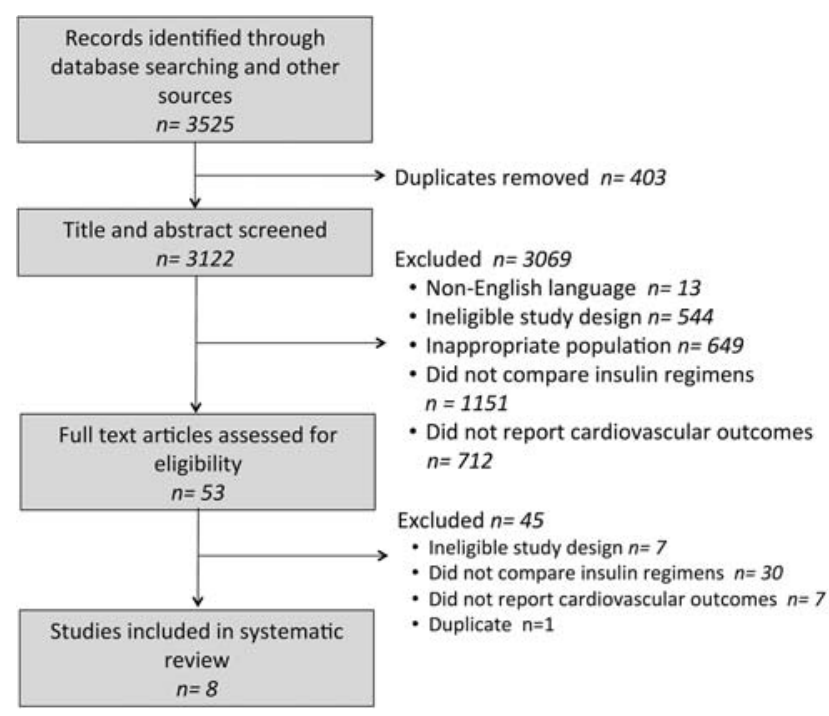

Figure 1 Selection of studies. 
$(n=30)$, or they did not report CV outcomes $(n=7)$, and there was one duplicate. The citations of excluded studies with their exclusion reasons can be found in online supplementary appendix B. Online supplementary appendices $\mathrm{C}$ and $\mathrm{D}$ contain the results of the risk of bias assessment for RCTs and cohort studies included in our systematic review. In summary, both RCTs were highly susceptible to bias as several domains within the Cochrane Risk of Bias tool indicated several sources of potential bias. Although the cohort studies scored relatively high on the Newcastle-Ottawa Scale, whereby all studies had six or more stars out of a maximum of nine stars, most studies did not demonstrate that the outcome of interest had not occurred prior to cohort entry for non-fatal CV events. Given many studies did not appear to validate that $\mathrm{CV}$ events had not occurred prior to insulin initiation, these studies may have included patients that experienced either an incident or recurrent CV event, which in turn would impact event risks and comparability among study results.

\section{Study characteristics}

This review summarises the data from eight studies (2 RCTs and 6 cohort studies) including a total 109910 patients. ${ }^{14-21}$ All of the included studies were designed to investigate $\mathrm{CV}$ outcomes in adults with type 2 diabetes using different insulin regimens (table 1 ). No two studies contrasted the same insulin exposures. Two RCTs, the University Group Diabetes Program (UGDP) trial $^{15}$ and the Hyperglycaemia and Its Effects After Acute Myocardial Infarction on Cardiovascular Outcomes in Patients with Type 2 Diabetes Mellitus (HEART2D) trial, ${ }^{14}$ met the inclusion criteria for this systematic review.

The UGDP trial was an open-label, prospective, five-armed, RCT that was designed in 1961 to evaluate the effects of different hypoglycaemic treatments on vascular disease in patients with type 2 diabetes. The trial consisted of a fixed dose insulin arm (U-80 Lente Iletin insulin, $10,12,14$ or 16 units/day based on body surface), a variable dose insulin arm (U-80 Lente Iletin insulin or other insulins, as much as required to maintain 'normal' glucose control, minimum 5 units/day), two oral agent arms (tolbutamide $1.5 \mathrm{~g}$ /day or phenfor$\min 100 \mathrm{mg} /$ day) and a placebo control arm. There were 210 patients randomised to the fixed insulin group and 204 randomised to the variable insulin group. The mean age of all patients randomised was 53 years, $71 \%$ were female and $46 \%$ had a history of CV disease at baseline. $^{22}$

The HEART2D was an open-label, prospective, two-armed, RCT that began in 2002 with the aim of comparing the effects of prandial versus fasting glycaemic control on risk of macrovascular outcomes in patients with type 2 diabetes after acute MI. Patients with type 2 diabetes were recruited within 21 days of an acute MI and randomised to either a prandial insulin regimen (premeal insulin lispro three times per day and basal neutral protamine hagedorn (NPH) insulin at bedtime if needed) or a basal insulin regimen (NPH insulin two times per day or insulin glargine once daily) targeting a $2 \mathrm{~h}$ postprandial blood glucose $<7.5 \mathrm{mmol} / \mathrm{L}$ and a fasting plasma glucose $<6.7 \mathrm{mmol} / \mathrm{L}$, respectively. A total of 1115 patients underwent randomisation and mean length of follow-up was 32.4 months. At baseline, the mean age was 61 years, $37 \%$ were female and patients had been living with diabetes for 9 years prior to the trial initiation. ${ }^{14}$

Included cohort studies identified study cohorts of patients with type 2 diabetes using secondary data sources, either administrative claims databases or electronic medical records, and compared the incidence of CV outcomes in patients using different insulin regimens. Each cohort study evaluated different insulin-exposure contrasts. All of the cohort studies were conducted between 1981 and 2011 and had average length of follow-up between 6 and 61.2 months.

Briefly, using the administrative claims databases of Saskatchewan Health (Canada), Gamble $e t a l^{16}$ compared all-cause and CV mortality between patients exposed to no, low, moderate and high amounts of insulin among 12272 new users of antidiabetic medications. Patients were on average 65 years of age, $45 \%$ were female and the mean follow-up time was 5.1 years. Hall $e t a l^{17}$ used the UK-based The Health Improvement Network (THIN) primary care database (electronic medical records) to estimate the risk of vascular disease between initiators of different insulins among 3485 patients with type 2 diabetes who had inadequate control on either 2 or 3 oral agents. Patients were on average 62 years of age, $41 \%$ were female and the mean follow-up time was 3.6 years. Juhaeri et $a l^{18}$ identified 65619 patients with type 2 diabetes from the US-based PharMetrics claims database (administrative records) and compared the incidence rate of major vascular outcomes in patients taking insulin glargine compared to other insulin regimens. ${ }^{18}$ The majority of patients were 60 years of age or less $(68 \%), 51 \%$ were female and the follow-up time was not reported. Kress et $a l^{19}$ compared the incidence of microvascular and macrovascular outcomes in patients with type 2 diabetes ( $\mathrm{n}=12109$; average age 64 years; $47 \%$ were female; average follow-up of 3.5 years) treated with insulin glulisine versus regular human insulin using a German-based IMS Disease Analyzer database (electronic medical records). The same database was used in a study by Rathmann and Kostev ${ }^{20}$ to evaluate the incidence of microvascular and macrovascular outcomes in patients with type 2 diabetes $(n=6308$; average age 60 years; $43 \%$ were female; average follow-up of 3.5 years) using insulin aspart versus regular human insulin. Finally, a cohort study by Rhoads $e t a l^{21}$ compared the incidence of an acute MI in new users $(\mathrm{n}=20191$; average age 56 years; $46 \%$ were female; average follow-up of 2 years) of insulin glargine versus NPH insulin using the US-based Integrated Health Care Information Systems administrative claims database. 


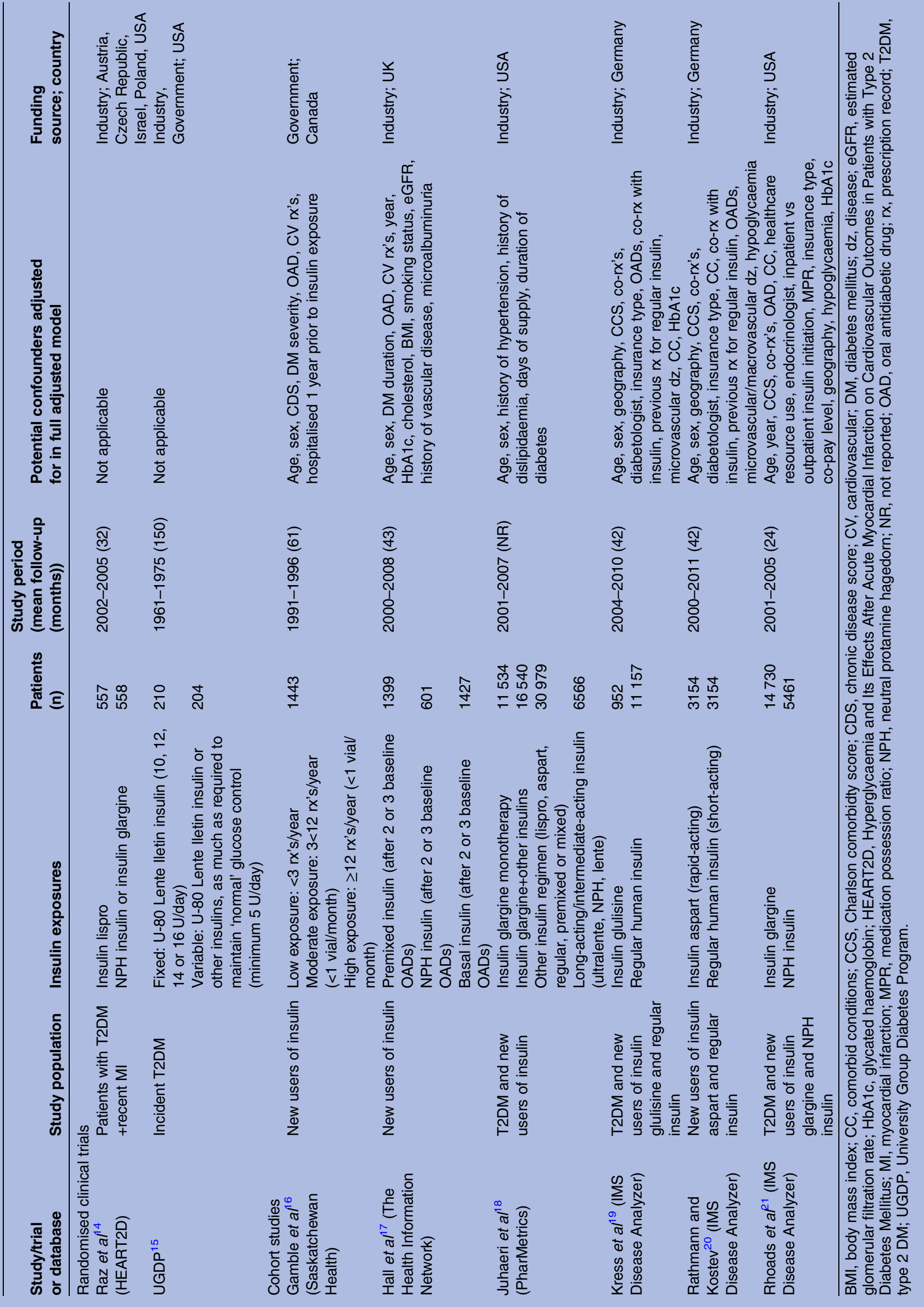




\section{CV outcomes}

There were no statistically significant differences in CV outcomes reported in the HEART2D trial or the UGDP study (figure 2). A total of 210 fatal and non-fatal MIs were reported among 1529 patients. The HEART2D trial reported no differences in the risk of non-fatal MI ( $\mathrm{RR}=1.06,95 \%$ CI 0.73 to 1.53$)$, fatal $\mathrm{MI}(\mathrm{RR}=1.00,95 \%$ CI 0.45 to 2.21), and non-fatal or fatal $\mathrm{MI}(\mathrm{RR}=1.00$, $95 \%$ CI 0.72 to 1.39$)$. The UGDP study reported similar results for the risk of a fatal or non-fatal MI $(\mathrm{RR}=1.03$, $95 \%$ CI 0.70 to 1.51$)$, non-fatal MI ( $R R=1.00,95 \%$ CI 0.62 to 1.60$)$ and fatal MI alone ( $R R=1.12,95 \%$ CI 0.52 to 2.39$)$.

The HEART2D trial also reported the number of patients who experienced a stroke $(n=37)$ but again found no between-group differences in the risk of a nonfatal stroke ( $R R=1.19,95 \%$ CI 0.62 to 2.29$)$, fatal stroke $(\mathrm{RR}=1.50,95 \% \mathrm{CI} 0.25$ to 8.96$)$, and non-fatal or fatal stroke ( $R R=1.18,95 \%$ CI 0.62 to 2.23). CV death was reported by both RCTs. This included a total of 86 CV-related deaths that occurred in the HEART2D trial and $63 \mathrm{CV}$-related deaths that occurred in the UGDP study. The RR of CV death in the HEART2D trial was 1.05 (95\% CI 0.70 to 1.58 ) and 1.00 (95\% CI 0.63 to 1.57) in the UGDP trial.

The cohort studies reported statistically significant and non-significant relative differences in $\mathrm{CV}$ outcomes among various insulin regimens (figure 3 ). The risk of a non-fatal or fatal MI was reported by four cohort studies $^{18-21}$ and included six different insulin-exposure contrasts. Adjusted point estimates reported for two of the insulin contrasts indicated a statistically significant difference in the risk of a non-fatal or fatal MI: insulin aspart versus regular insulin ( $\mathrm{HR}=0.69,95 \%$ CI 0.54 to 0.88 ) and NPH insulin versus other basal insulin regimens (HR=1.39, 95\% CI 1.14 to 1.69 ).

Stroke outcomes were reported by three cohort studies. ${ }^{18-20}$ Fatal+non-fatal stroke was evaluated for five different insulin-exposure contrasts of which two exposure contrasts indicated risk differences in fatal+non-fatal stroke: other insulin regimen versus long-acting/ intermediate-acting insulin ( $\mathrm{HR}=1.20,95 \%$ CI 1.04 to 1.40 ) and insulin aspart versus regular human insulin (HR $=0.58,95 \%$ CI 0.45 to 0.74$)$.

MACE outcomes were reported by one cohort study ${ }^{17}$ that included adjusted point estimates for four different insulin regimen comparisons. Hall and colleagues did not report any statistically significant difference in the risk of MACE among users of different insulin regimens (figure 3).

\section{All-cause mortality}

There were no statistically significant differences in allcause mortality reported in the HEART2D trial $(\mathrm{RR}=1.00,95 \%$ CI 0.69 to 1.45$)$ or the UGDP study $(\mathrm{RR}=1.03,95 \%$ CI 0.74 to 1.44 ; figure 2$)$. Only one cohort study, Gamble and colleagues, included all-cause mortality as an outcome of interest. They found a doseresponse relationship whereby more insulin exposure was associated with a higher risk of mortality (figure 3).
Figure 2 Unadjusted relative risk $(95 \% \mathrm{Cl})$ of cardiovascular outcomes and all-cause mortality using different insulin regimens calculated based on number of events from included RCTs (MI, myocardial infarction; RCTs, randomised controlled trials; UGDP, University Group Diabetes Program).

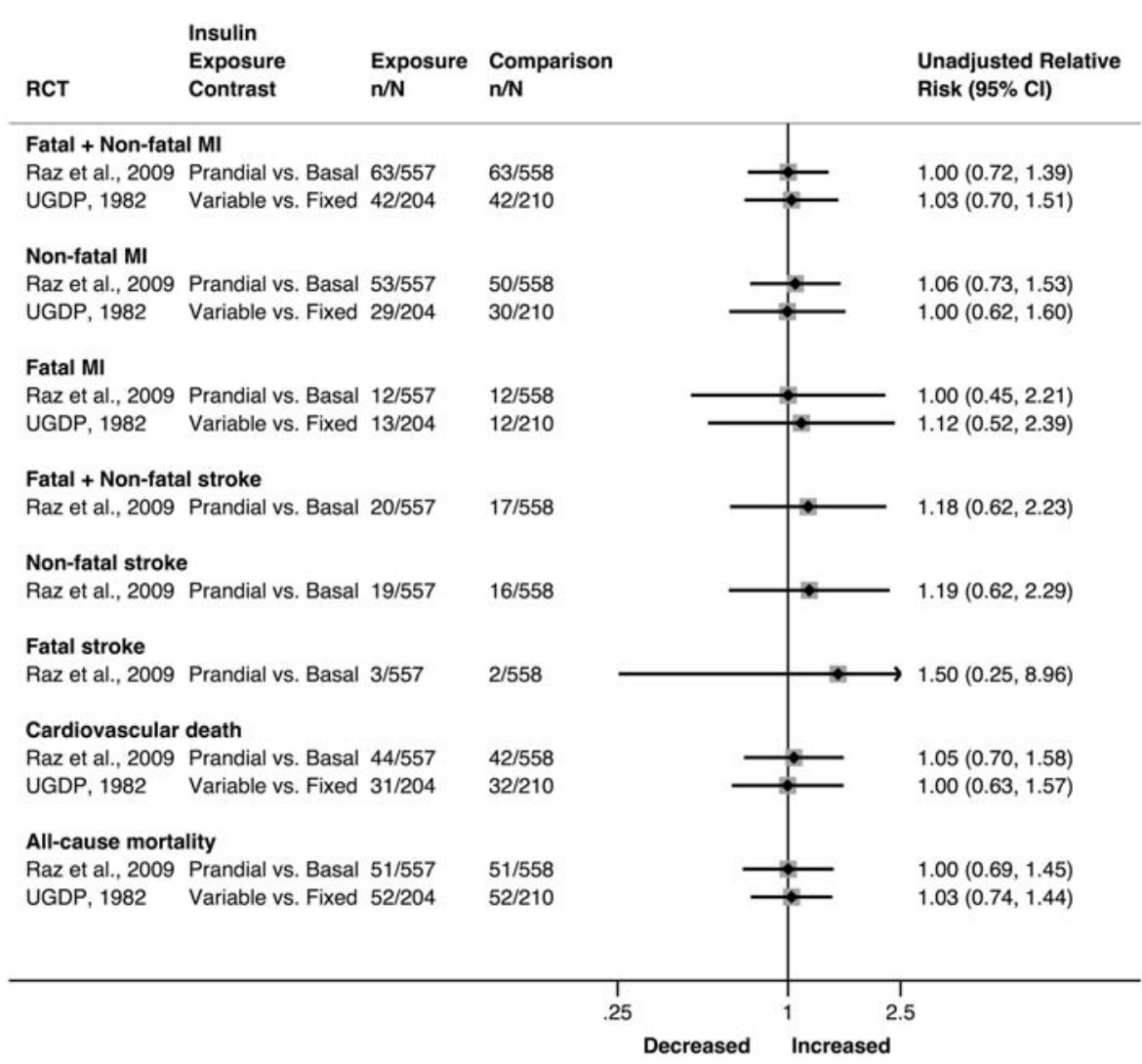




\section{DISCUSSION}

Our summary of the available literature indicates that there is a need for more high-quality evidence investigating the safety and effectiveness of different insulin regimens in patients with type 2 diabetes. Indeed, this systematic review documents a lack of high-quality evidence examining CV outcomes of different insulin regimens used to treat patients with type 2 diabetes. We identified eight studies, two RCTs and six cohort studies, which were specifically designed to evaluate differences in $\mathrm{CV}$ outcomes between insulin regimens. These eight studies were disparate in design and execution, whereby none of the studies examined CV risk across identical exposure categories. This high degree of clinical heterogeneity precluded the use of meta-analytic techniques to summarise a common treatment effect. Therefore, our systematic review descriptively summarised the results from each study. We found no clear pattern of harm or benefit for any particular insulin regimen. Most of the study findings suggested that no substantive differences in $\mathrm{CV}$ risk among insulin regimens exist; however, clinically important harms and benefits cannot be ruled out given the wide CIs reported by the RCTs and cohort studies.

Although several point estimates are suggestive of clinically important differences among different insulin regimens, most of the results from included studies do not suggest large differences among insulin regimens with respect to CV outcomes. Results from the included RCTs must be interpreted with caution given their susceptibility to bias and lack of generalisability to today's type 2 diabetes population. For example, treatment algorithms for type 2 diabetes and insulin formulations have changed substantially since the UGDP was completed over 30 years ago. Furthermore, the HEART2D trial was conducted in a specific high-risk post-MI population.

In the absence of high-quality RCTs, well-designed cohort studies may inform drug-outcome associations. Indeed, cohort studies provide a real-world assessment of insulin use and its relation with CV disease providing a high level of external validity. Our systematic review included cohort studies of varying susceptibility to bias with the majority found to have a low overall risk of bias. Nonetheless, cohort studies are susceptible to the

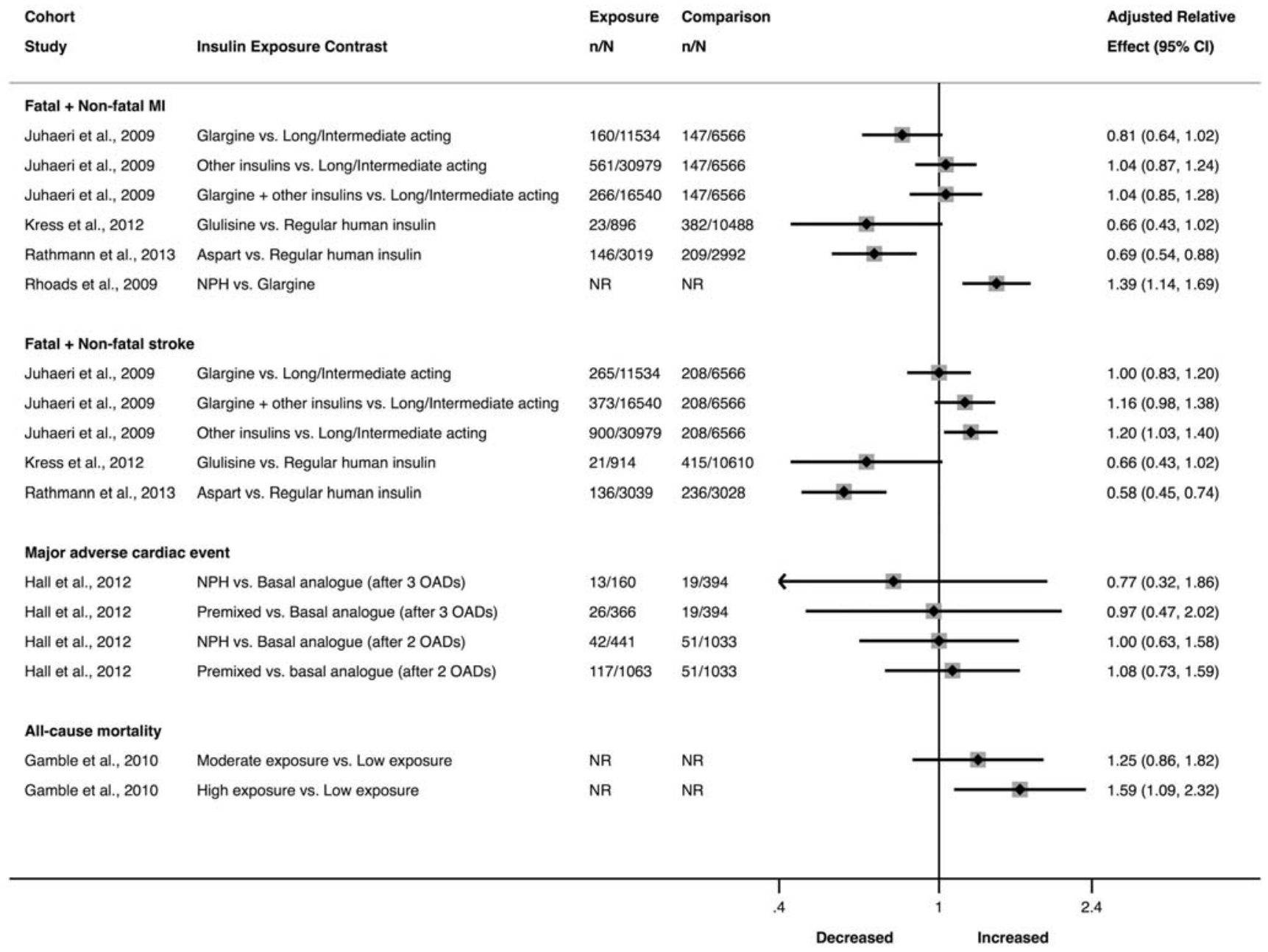

Figure 3 Point estimates $(95 \% \mathrm{Cl})$ of cardiovascular outcomes and all-cause mortality using different insulin regimens reported by included cohort studies (MI, myocardial infarction; NPH, neutral protamine hagedorn; OADs, oral antidiabetic drugs). 
disadvantages inherent in non-randomised studies, notably residual confounding and selection bias. For example, selection bias among insulin users is highly likely given insulin is often used when other antidiabetic medications provide inadequate glycaemic control. In fact, it is plausible that certain patients may be preferentially prescribed (ie, selected) with certain insulin regimens given specific clinical characteristics that are related to CV outcomes, such as frequency and severity of hypoglycaemia, postprandial hyperglycaemia or drug formulary restrictions, among others. However, all cohort studies used methods to reduce bias including restricting to new users of insulin, used active comparators, and used a population-based data set. Moreover, all cohort studies adjusted for multiple potential confounding variables with three cohort studies adjusting for glycaemic control (table 1). ${ }^{17} 19{ }^{21}$ Nonetheless there was variation in how these methods were applied and how covariates were entered into the statistical model. Although all of the cohort studies had a new user design, some of the studies allowed previous use of insulin that was not part of the exposure contrast of interest.

Continued research surrounding the real-world safety and effectiveness of insulin is important given its increasing utilisation among people with type 2 diabetes and potential safety concerns in this population. One potential safety concern surrounding insulin use in type 2 diabetes is the effect of exogenous insulin on the CV system. Some studies suggest that exogenous insulin may be cardioprotective and advocate that insulin therapy should be used early in the diagnosis of type 2 diabetes to relieve symptomatic hyperglycaemia, ${ }^{23-26}$ preserve $\beta$-cell function ${ }^{27}$ and prevent microvascular complications. ${ }^{28}$ However, there is also compelling evidence that hyperinsulinaemia may negatively impact CV outcomes in patients with type 2 diabetes. ${ }^{929-35}$ Supraphysiological insulin levels following high doses of insulin therapy in the context of insulin resistance is thought to create a proinflammatory, mitogenic environment within the vascular system. ${ }^{30} 36$ Furthermore, several large observational cohort studies have found patients exposed to insulin to have a higher risk of CV and all-cause mortality compared to oral antidiabetic therapy. ${ }^{16} \quad 31 \quad 37$ Moreover, the results of several large randomised clinical trials have either showed harm or failed to show improvement in macrovascular outcomes with intensive glycaemic strategies that by necessity involved intensive insulin therapy. ${ }^{38-42}$ These studies were not included in our systematic review as they did not have an insulin comparator group.

To the best of our knowledge this systematic review was the first study to summarise the evidence generated from RCTs and observational studies comparing the incidence of CV outcomes in patients with type 2 diabetes using different types, doses and/or regimens of insulin. Strengths of this review include that it followed a published protocol that included a prespecified search strategy, eligibility criteria, data extraction plan, outcome specification and analysis plan. A risk of bias assessment was performed for each included study, the results of which guided data interpretation and analysis. Unfortunately, we were unable to quantitatively pool the data due to the extremely high degree of clinical heterogeneity. We also did not assess publication bias due to the small number of studies included, which again were heterogeneous in their exposure groups. Further limitations include the lack of a search strategy to explicitly identify grey literature. We decided to focus the search strategy on biomedical databases and the reference lists of relevant studies. Non-English language papers were excluded from this review; however, previous literature suggests that language restrictions do not impact the findings of systematic reviews. ${ }^{43} 44$

Owing to the limited number of patients and outcome events, and heterogeneous study designs, more highquality evidence is needed to determine if clinically significant differences exist between insulin regimens with respect to $\mathrm{CV}$ outcomes in patients with type 2 diabetes. Ideally, well-executed and blinded pragmatic RCTs that allocate patients with type 2 diabetes to different insulin regimens and evaluate $\mathrm{CV}$ outcomes would provide the highest quality information to elucidate this relationship. In their absence, well-designed observational studies may further define the potential CV risks or benefits for patients with type 2 diabetes using different insulin regimens.

Contributors HIP and J-MG were involved in the concept and design of the study. HIP was responsible for coauthoring the protocol, conducting the literature search and screening, performing the risk of bias assessments, extracting and analysing the data, and preparation of the first version of the manuscript. MDA acted as second reviewer during the literature screening, risk of bias assessment and data extraction steps. J-MG originally conceived the study idea, coauthored the protocol, was responsible for overseeing all aspects of the study, contributed to the interpretation of study results, and coauthored the manuscript. All authors provided critical revisions to the manuscript and have approved the final manuscript.

Funding This study was supported by the Newfoundland and Labrador Research and Development Corporation. J-MG is supported by a New Investigator Award from the Canadian Institutes of Health Research and a Clinician Scientist Award from the Canadian Diabetes Association.

Competing interests None.

Provenance and peer review Not commissioned; externally peer reviewed.

Data sharing statement No additional data are available.

Open Access This is an Open Access article distributed in accordance with the Creative Commons Attribution Non Commercial (CC BY-NC 4.0) license, which permits others to distribute, remix, adapt, build upon this work noncommercially, and license their derivative works on different terms, provided the original work is properly cited and the use is non-commercial. See: http:// creativecommons.org/licenses/by-nc/4.0/

\section{REFERENCES}

1. Canadian Diabetes Association Clinical Practice Guidelines Expert Committee. Canadian Diabetes Association 2013 Clinical Practice Guidelines for the Prevention and Management of Diabetes in Canada. Can J Diabetes 2013;37:S1-212.

2. American Diabetes Association. Standards of medical care in diabetes-2014. Diabetes Care 2013;37:S14-80. 
3. Filion KB, Joseph L, Boivin J-F, et al. Trends in the prescription of anti-diabetic medications in the United Kingdom: a population-based analysis. Pharmacoepidemiol Drug Saf 2009;18:973-6.

4. Centers for Disease Control and Prevention. National Diabetes Statistics Report: estimates of diabetes and its burden in the United States, 2014. http://www.cdc.gov/diabetes/pubs/statsreport14/ national-diabetes-report-web.pdf (accessed 15 Jul 2014).

5. Ringborg A, Lindgren $\mathrm{P}$, Yin DD, et al. Time to insulin treatment and factors associated with insulin prescription in Swedish patients with type 2 diabetes. Diabetes Metab 2010;36:198-203.

6. Canadian Agency for Drugs and Techologies in Health (CADTH). Current Utilization of Insulin Products in Canada. Canadian Optimal Medication Prescribing and Utilization Service (COMPUS), 2008. http://www.cadth.ca/media/pdf/compus_Current-Utilization-Report.pdf (accessed 15 Jul 2014).

7. Leiter LA, Berard L, Bowering CK, et al. Type 2 diabetes mellitus management in Canada: is it improving? Can J Diabetes 2013;37:82-9.

8. Boussageon R, Gamble JM, Gueyffier F, et al. Clinically relevant efficacy of insulin therapy in patients with type 2 diabetes. Therapie 2013;68:415-17.

9. Currie CJ, Johnson JA. The safety profile of exogenous insulin in people with type 2 diabetes: justification for concern. Diabetes Obes Metab 2012;14:1-4.

10. Morrish NJ, Wang SL, Stevens LK, et al. Mortality and causes of death in the WHO multinational study of vascular disease in diabetes. Diabetologia 2001:44(Suppl 2):S14-21.

11. Price HI, Agnew M, Gamble JM. Comparative cardiovascular morbidity and mortality in patients taking different insulin regimens for type 2 diabetes: a systematic review. PROSPERO 2014: CRD42014007631. http://wwwcrdyorkacuk/PROSPERO/display_ recordasp?|D=CRD42014007631.

12. Cochrane Handbook for Systematic Reviews of Interventions. Version 5.1.0 [updated March 2011] ed: The Cochrane Collaboration, 2011.

13. Wells GA, Shea B, O'Connell D, et al. The Newcastle-Ottawa Scale (NOS) for assessing the quality of nonrandomised studies in meta-analyses. Secondary The Newcastle-Ottawa Scale (NOS) for assessing the quality of nonrandomised studies in meta-analyses, 2014. http://www.ohri.ca/programs/clinical_epidemiology/oxford.asp

14. Raz I, Wilson PW, Strojek K, et al. Effects of prandial versus fasting glycemia on cardiovascular outcomes in type 2 diabetes: the HEART2D trial. Diabetes Care 2009;32:381-6.

15. Knatterud GL, Klimt CR, Goldner MG, et al.; University Group Diabetes Program. Effects of hypoglycemic agents on vascular complications in patients with adult-onset diabetes VIII. Evaluation of insulin therapy: final report. Diabetes 1982;31:1-25.

16. Gamble J-M, Simpson SH, Eurich DT, et al. Insulin use and increased risk of mortality in type 2 diabetes: a cohort study. Diabetes Obes Metab 2010;12:47-53.

17. Hall GC, McMahon AD, Carroll D, et al. Observational study of the association of first insulin type in uncontrolled type 2 diabetes with macrovascular and microvascular disease. PLOS ONE 2012;7:e49908.

18. Juhaeri J, Gao S, Dai WS. Incidence rates of heart failure, stroke, and acute myocardial infarction among type 2 diabetic patients using insulin glargine and other insulin. Pharmacoepidemiol Drug Saf 2009;18:497-503.

19. Kress S, Kostev K, Dippel FW, et al. Micro- and macrovascular outcomes in type 2 diabetic patients treated with insulin glulisine or human regular insulin: a retrospective database analysis. Int J Clin Pharmacol Ther 2012;50:821-9.

20. Rathmann W, Kostev K. Lower incidence of recorded cardiovascular outcomes in patients with type 2 diabetes using insulin aspart vs. those on human regular insulin: observational evidence from general practices. Diabetes Obes Metab 2013;15:358-63.

21. Rhoads GG, Kosiborod M, Nesto RW, et al. Comparison of incidence of acute myocardial infarction in patients with type 2 diabetes mellitus following initiation of neutral protamine Hagedorn insulin versus insulin glargine. Am J Cardiol 2009;104:910-16.

22. Klimt CR, Knatterud GL, Meinert CL, et al. A study of the effects of hypoglycemic agents on vascular complications in patients with adult-onset diabetes: I. Design, methods, and baseline results. Diabetes 1970;19:747-83.
23. Chaudhuri A, Dandona P, Fonseca V. Cardiovascular benefits of exogenous insulin. J Clin Endocrinol Metab 2012;97:3079-91.

24. Bretzel RG. Intensive insulin regimens: evidence for benefit. Int $J$ Obes Relat Metab Disord 2004;28(Suppl 2):S8-13.

25. Dandona P, Aljada A, Mohanty P. The anti-inflammatory and potential anti-atherogenic effect of insulin: a new paradigm. Diabetologia 2002;45:924-30.

26. Engel-Nitz NM, Martin S, Sun P, et al. Cardiovascular events and insulin therapy: a retrospective cohort analysis. Diabetes Res Clin Pract 2008;81:97-104.

27. Kumar A. Early use of insulin for beta cell preservation. J Assoc Physicians India 2007;55(Suppl):26-8.

28. Stratton IM, Adler Al, Neil HA, et al. Association of glycaemia with macrovascular and microvascular complications of type 2 diabetes (UKPDS 35): prospective observational study. BMJ 2000;321:405-12.

29. Rensing KL, Reuwer $\mathrm{AQ}$, Arsenault BJ, et al. Reducing cardiovascular disease risk in patients with type 2 diabetes and concomitant macrovascular disease: can insulin be too much of a good thing? Diabetes Obes Metab 2011;13:1073-87

30. Nandish S, Bailon O, Wyatt J, et al. Vasculotoxic effects of insulin and its role in atherosclerosis: what is the evidence? Curr Atheroscler Rep 2011;13:123-8.

31. Currie CJ, Poole CD, Evans M, et al. Mortality and other importan diabetes-related outcomes with insulin vs other antihyperglycemic therapies in type 2 diabetes. J Clin Endocrinol Metab 2013:98:668-77.

32. Muis MJ, Bots ML, Grobbee DE, et al. Insulin treatment and cardiovascular disease; friend or foe? A point of view. Diabetic Med 2005;22:118-26.

33. Lebovitz HE. Insulin: potential negative consequences of early routine use in patients with type 2 diabetes. Diabetes Care 2011;34: S225-30.

34. Janka HU, Standl E. Hyperinsulinaemia as a possible risk factor of macrovascular disease in diabetes mellitus. An overview. Diabete Metab 1987;13:279-83.

35. Margolis DJ, Hoffstad O, Strom BL. Association between serious ischemic cardiac outcomes and medications used to treat diabetes. Pharmacoepidemiol Drug Saf 2008;17:753-9.

36. Draznin B. Mitogenic action of insulin: friend, foe or 'frenemy'? Diabetologia 2010;53:229-33.

37. Roumie CL, Greevy RA, Grijalva CG, et al. Association between intensification of metformin treatment with insulin vs sulfonylureas and cardiovascular events and all-cause mortality among patients with diabetes. JAMA 2014;311:2288-96.

38. Patel A, MacMahon S, Chalmers J, et al. Advance Collaborative Group. Intensive blood glucose control and vascular outcomes in patients with type 2 diabetes. N Engl $J$ Med 2008;358:2560-72.

39. Gerstein HC, Miller ME, Byington RP, et al; Action to Control Cardiovascular Risk in Diabetes Study Group. Effects of intensive glucose lowering in type 2 diabetes. N Engl J Med 2008;358:2545-59.

40. Gerstein HC, Bosch J, Dagenais GR, et al. Origin Trial Investigators. Basal insulin and cardiovascular and other outcomes in dysglycemia. N Engl J Med 2012;367:319-28.

41. Duckworth W, Abraira C, Moritz T, et al. Glucose control and vascular complications in veterans with type 2 diabetes. $N$ Engl $J$ Med 2009;360:129-39.

42. Schernthaner G. Diabetes and cardiovascular disease: is intensive glucose control beneficial or deadly? Lessons from ACCORD, ADVANCE, VADT, UKPDS, PROactive, and NICE-SUGAR. Wien Med Wochenschr 2010;160:8-19.

43. Morrison A, Polisena J, Husereau D, et al. The effect of English-language restriction on systematic review-based meta-analyses: a systematic review of empirical studies. Int $J$ Technol Assess Health Care 2012;28:138-44.

44. Moher D, Klassen TP, Schulz KF, et al. What contributions do languages other than English make on the results of meta-analyses? J Clin Epidemiol 2000;53:964-72. 